\title{
Recovery of Skeletal Striated Muscle Tissue in a Model of Reversible Injury
}

\section{Nicolas Salazar and Doris Haydee Rosero*}

Universidad Icesi, Cali, Valle del Cauca, Colombia

"Corresponding author: Doris Haydee Rosero-Salazar, Department of Medical Basic Sciences, Universidad Icesi, Cali, Valle del Cauca, Colombia, Tel: +573183761429; E-mail: dorhaross@gmail.com

Received date: April 30, 2017; Accepted date: August 01, 2017; Published date: August 08, 2017

Copyright: (C) 2017 Salazar N, et al. This is an open-access article distributed under the terms of the Creative Commons Attribution License, which permits unrestricted use, distribution and reproduction in any medium, provided the original author and source are credited.

\begin{abstract}
Reversible injury is a dynamic condition in tissues exposed to multiple harm situations including ischemia and post-ischemic recovery, in which the skeletal striated muscle tissue evidences histopathological characteristics to adapt to damage. During the inflammation and the regeneration phases of healing, the myocytes show morphological changes with an apparently complete recovery at the end of the treatment. Though the satellite cells play a key role in the recovery, not in all cases the regeneration is completely achieved. That is why in this research we measured the histopathological patterns evidenced by enzyme histochemistry and morphometric measurements, during the spontaneous recovery of skeletal muscle fibers underwent to short periods of ischemia of one and three hours and prolonged periods of reperfusion up to 32 days (768 hours). The selected muscles were the extensor carpi radialis longus and the soleus from Wistar rats.

There were significant differences in the distribution of type of fibers, shape, size, leukocyte infiltration, necrosis and presence of central nuclei. The soleus muscle adapts better during early reperfusion than extensor carpi radialis longus does. Nevertheless, both muscles evidenced an incomplete recovery at day 32 . The extrapolation of these findings suggests the importance of further following up of patients and the improvement of recovery the muscle function after surgery and/or other harm conditions.
\end{abstract}

Keywords: Ischemia; Reperfusion; Morphometry; Myocytes; Skeletal muscle

\section{Introduction}

Orthopedic surgeries such as arthroscopic procedures imply the use of a pneumatic or a manual tourniquet. Also, controlling bleeding due to accidents occurring in distant places and treatments for joint diseases or contractures requires a tourniquet. The use of the tourniquet entails reversible injury triggered by ischemic conditions during determined periods of time [1-3]. Arthroscopic procedures, for instance, are performed during 15 to 60 minutes, while to transport an injured person from far places to the nearest health institution, can require two to three hours.

In those cases, the muscles under the level of compression suffer the associated damage due to the ischemic condition followed by reperfusion injury [4,5]. The lesion patterns studied in myocytes after induced ischemia of two to four hours comprise edema, atrophy and changes in their surrounding connective tissue [5-8]. The recovery of the histological and functional characteristics of the muscles depends on their extracellular matrix and the type of fibers. The molecular mechanisms of adaptation lead to muscle fibers to change their oxidative or glycolytic predominance in the muscle $[4,9,10]$.

More than two decades ago, it was considered that irreversible injury mainly occurs during ischemic conditions longer than five hours $[11,12]$. However, since the last 20 years, the evidence has pointed out that ischemic conditions less than three hours caused by an acute compartmental syndrome, tourniquet or artery clamp, for example, lead to necrosis $[3,13]$. The stem cells of the skeletal striated muscle tissue, called satellite cells, are postnatal myogenic precursors that can regenerate the myocytes [14]. Then, the recovery of the muscle tissue underwent to reversible injury should lead to a proper rearrangement spontaneously. Some studies regarding a complete recovery of skeletal muscle include the injection of stromal mesenchymal cells into the injured muscle, in which the myocytes achieve a complete recovery 14 days after reperfusion [15]. This is an important aspect to consider for treatment after injury. A different research based on mouse models evidenced an apparently total functional recovery by day 56 after the ischemic condition. This was performed with a manual tourniquet without control of the pressure of ischemia [16].

In the present study, through conventional and enzymatic histochemistry, were analyzed the changes of lesion and recovery caused by an induced ischemic injury in skeletal muscle fibers. Treatments or additional interventions were not applied to describe the histopathological characteristics of the spontaneous recovery and/or adaptation in the skeletal striated muscle tissue throughout postischemic lesion until day 32 .

\section{Materials and Methods}

\section{Experimental animals}

All procedures performed during this research involving lab animals, were in accordance with the ethical standards of the Animal Ethics Committee of Universidad del Valle and International Guidelines for Care and Use of Animals [17]. The Ethics Committee approved this research through the act 036-15. This study was 
conducted by using 42 male adult rats, Wistar albino strain, genus Rattus, 12 months old and weighing 450 to 500 grams.

The rats were obtained from the bioterium of the Universidad del Valle, which provided to the animals with food and water ad libitum. These rats were handled considering the Institutional Standard Guides to the Use of Laboratory Animals. The extensor carpi radialis longus and soleus were suitable muscles for this research, due to their glycolytic and oxidative predominance of their muscle fibers respectively $[4,10,18]$. The surgical access is relatively easy, mainly for the forelimb. This let us to reduce the surgical time required for the dissection of both muscles, important for a proper histochemical staining.

\section{Surgical procedure}

The rats were divided into one control group and 20 experimental groups of ischemia of one hour and three hours followed by reperfusion of 0 hours, 1 hour, 16 hours, 24 hours, 2 days (48 hours), 4 days (96 hours), 8 days (192 hours), 16 days (384 hours), 24 days (576 hours) and 32 days (768 hours), respectively. These periods of reperfusion were selected based on previous studies focused on morphological and physiological recovery of the skeletal muscle tissue after ischemic injury $[4,5,19,20]$. The total sample size of 42 rats was determined based on 21 groups, significant level of 0.05 and confident level of 0.95 .

Under anesthetic condition with Isofluorane ${ }^{\odot}$ Baxter and pentobarbital (Penthal ${ }^{\circ}$ - INVET $64.8 \mathrm{mg} / \mathrm{ml}$ ) 4-5 mg/100 grams per weight, two pneumatic tourniquets were set to each rat. One tourniquet was set in the proximal region of the right forelimb near to the shoulder, and the other one in the proximal region of the left hindlimb near to the hip. The complete description of both tourniquets can be founded in our previous work [8]. The pressure of ischemia was adjusted at $260 \mathrm{mmHg}$ during one or three hours regarding to each experimental group. This pressure is suitable to perform a complete ischemic condition; due to microcirculatory blood flow is not present at $230 \mathrm{mmHg} \pm 20 \mathrm{mmHg}[1,4,15,21]$. Once the time of ischemia finished, both tourniquets were removed. Postsurgical analgesia with tramadol $2 \mathrm{mg} / 100 \mathrm{mg}$ was administered to each rat.

\section{Histochemical stains}

Once the time of reperfusion was over, an overdose of pentobarbital was injected intraperitoneally to perform euthanasia. During the following 5 to 10 minutes we verify the death signs (respiratory failure, absence of heart beat and opaque cornea) and subsequently we dissected soleus and extensor carpi radialis longus muscles. The samples were directly fixed by freezing using 2-methyl butane (isopentane) cooled in liquid nitrogen, stored at $-70^{\circ} \mathrm{C}$ and cut on cryostat Leica Jung Frigocut $2800 \mathrm{~N}$ at $-20^{\circ} \mathrm{C}$ and 16 ums. The stain techniques were enzyme histochemistry for NADH-TR (nicotinamide adenine dinucleotide tetrazolium reductase), mATPase $\mathrm{pH} 4.6$ (myosin adenosine triphosphatase), and conventional histochemistry for H\&E (hematoxylin and eosin).

For NADH-TR, the tissues were incubated during 60 minutes at $37^{\circ} \mathrm{C}$ in Tris $0.2 \mathrm{M}$ solution (Sigma-Aldrich ${ }^{\circ}$ ) pH 7.4 with $\mathrm{HCl} 0.1 \mathrm{M}$, Nitro-Blue Tetrazolium $10 \mathrm{mg}$ (Sigma-Aldrich ${ }^{\circledR}$ ) and Beta Nicotinamide Adenine dinucleotide $8 \mathrm{mg}$ (Sigma-Aldrich ${ }^{\odot}$ ) in distilled water $\mathrm{pH}$ 7.2. Oxidative fibers stained dark and glycolytic stained clearer. For mATPase stain, the samples were pre-incubated in ATP I during 25 minutes at $22^{\circ} \mathrm{C}$. Then, ATP II solution by using adenosine 5 'triphosphate (Sigma-Aldrich ${ }^{\oplus}$ ) $15 \mathrm{mg}$ was prepared, and adjusted to $\mathrm{pH}$ 10.6. The slides were incubated during 40 minutes at $37^{\circ} \mathrm{C}$ and fibers were revealed with ammonium sulfide $1 \%$ for 15 seconds. The acidic pre-incubation lead to a stronger reaction in the slow twitchfibers while fast twitch fibers stain pale [22,23].

\section{Morphometric analysis}

The oxidative- slow twitch fibers and glycolytic- fast twitch fibers were measured by image processing and segmentation of regions, to determine the areas of interest through image analysis techniques $[24,25]$. The other parameters measured through semiautomatic counting were:

- Leukocyte infiltration in muscle fibers

- Round shape

- Increasing in the size of myocytes

- Decreasing in the size of myocytes

- Presence of central nuclei

- Necrosis, evidenced by a pale stain, fragmentation of cytoplasm and loss of cellular morphology.

These patterns and measurements of the areas were performed by using Image Pro Plus v7.0 (Media Cybernetics Inc.). The measurements of each parameter were carried out to 174 images per muscle, randomly selected from a total amount of 404 microscopic fields in 40X magnification to determine cell characteristics. This size of images per muscle was calculated based on a confidence interval of 1.96. Statistical analysis consisted of comparisons between one and three hours of ischemia during reperfusion through Mann Whitney test. The periods of ischemia/reperfusion that showed significant differences were analyzed by multiple comparisons test, as nonparametric Bonferroni post-ANOVA. Kruskal - Wallis test was used for comparisons among the reperfusion groups, this is the nonparametric equivalent of the one-way analysis of variance. SPSS version 22 (IBM Analytics Corp.) was the statistical software used for this analysis.

This study described morphological characteristics of limb skeletal myocytes, during the recovery after short and prolonged periods of ischemic injury. The stain for NADH-TR lead to the identification of oxidative and glycolytic muscle fibers, while mATPase $\mathrm{pH} 4.6$ permits the recognition of slow twitch (type I) and fast twitch fibers (Type IIB). Both stain techniques complement each other in the analysis of the histopathological patterns observed in a muscle biopsy.

\section{Results}

The control group evidenced a normal predominance in the glycolytic fibers and fast-twitch fibers in the extensor carpi radialis longus muscle. In contrast, the soleus muscle has oxidative fibers predominance and a slow-twitch fibers majority (Table 1). For both muscles, the myocytes in the cross-sectional microscopic fields evidenced hexagonal-like shape and multiple nuclei adjacent to the sarcolemma. The above mentioned abnormal parameters in the morphometric analysis were negative for both control groups (Figures $1 \mathrm{~A}-1 \mathrm{C})$. 


\section{Extensor carpi radialis longus}

The experimental groups evidenced mainly during prolonged reperfusion, an increasing in the oxidative fibers areas in comparison to the control group. This characteristic suggests a change in the predominance of muscle fibers during reperfusion after being exposed to ischemic conditions of one hour and three hours p- value 0.045 (Figures 1D, 1G and 2A). The areas of slow twitch fibers were also superior in comparison to the control group, mainly during reperfusion after three hours of ischemia (Table 1). This tendency in the mechanism of contraction seen through mATPase $\mathrm{pH} 4.6$ suggests a functional adaptation during the recovery p-value 0.027 (Figures $1 \mathrm{E}$ and $3 \mathrm{~A})$.

In contrast, the areas of glycolytic fibers decreased during reperfusion after both periods of ischemia, which corresponds with the oxidative tendency pattern seen through NADH-TR p-value 0.034 (Figures 1D, 1G and 2A). To respect to the fast twitch fibers, this mechanism of contraction tended to increase during reperfusion after one hour of ischemia, which suggests that the muscle function can be conserved and recovered after short ischemic injuries (Table 1). During reperfusion after three hours of ischemia the total area of this type of fibers was minor but comparable the total area of the control group (Figures 1E and 3A).

Regarding to the other parameters measured through semiautomatic counting, the presence of leukocyte infiltration was a common characteristic in the surrounding connective tissue of the cells. Though it was present during reperfusion after both periods of ischemia, in this analysis we prefer to measure the presence of immune cells in the muscle fibers. This is important because these cells influence the recovery and/or scar tissue formation processes consistent with the severity of the injury. Leukocytes were mainly present during reperfusion after three hours of ischemia but during early reperfusion after one hour of ischemia p-value 0.025 . On the other hand, fibers with round shape were mainly observed during early periods of reperfusion after both periods of ischemia p-value 0.025 . The increasing in the size of muscle fibers was evidenced during prolonged reperfusion after three hours of ischemia p-value 0.025 . The decrease in the size of fibers was present during early reperfusion especially after the ischemic condition of three hours p-value 0.000 . Central nuclei were significant during prolonged reperfusion after one hour and three hours of ischemia, p-value 0.000 , while few necrotic fibers were present during early reperfusion after both periods of ischemia (Figures 1F, 1I and 4).

\section{Soleus}

The morphometric analysis evidenced a decrease of the oxidative predominance during reperfusion, mainly throughout reperfusion after three hours of ischemia, p-value 0.026. During reperfusion after one hour of ischemia, the amount of oxidative fibers was major or near to the control group areas (Figures $1 \mathrm{M}, 1 \mathrm{P}$ and $2 \mathrm{~B}$ ). The slow twitch fibers evidenced increases along reperfusion after one and three hours of ischemia p-value 0.032 (Figures $1 \mathrm{~N}, 1 \mathrm{Q}$ and $3 \mathrm{~B}$ ). These changes suggest that the soleus muscle adapts well to reperfusion injury to maintain its function and mechanism of contraction (Table 1).

The areas of glycolytic fibers were mainly present during prolonged reperfusion after the ischemic conditions of one and three hours, $\mathrm{p}$ value 0.027 (Figures $1 \mathrm{M}$ and $1 \mathrm{P}$ ). These areas did not exceed the areas measured for oxidative fibers. Likewise, a low quantity of areas of fast twitch fibers was observed throughout reperfusion after one and three hours of ischemia (Table 1). This characteristic suggests adaptation mechanisms of the soleus muscle to tolerate the ischemic injuries (Figures 1N, 1Q and 2B).

Regarding the other histopathological patterns, the presence of leukocyte infiltration was mainly observed during reperfusion after three hours of ischemia p-value 0.001. Round shape fibers were observed throughout reperfusion after one hour of ischemia and were mainly present during prolonged reperfusion after three hours of ischemia p-value 0.000 . In contrast, the increasing in the size of fibers was observed during prolonged reperfusion after one hour of ischemia p-value 0.022 , while few muscle fibers with decreasing on their size were observed in the microscopic fields. Central nuclei were not a significant change during reperfusion after one hour, but after three hours of ischemia these cells were still present during prolonged reperfusion p-value 0.001. Finally, few necrotic fibers were present during reperfusion after one and three hours of ischemia (Figures 1O, $1 \mathrm{R}$ and 5).

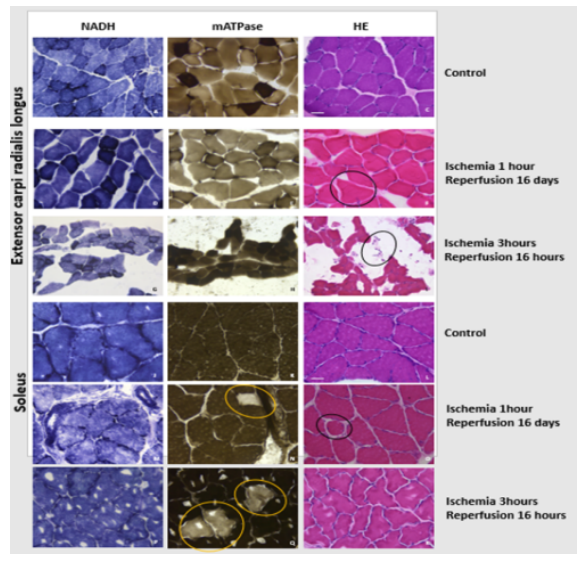

Figure 1: Parameters measured in muscle fibers during reperfusión. A-B-C Control group. Normal characteristics in the extensor carpi radialis longus observed on each staining. Glycolytic (NADH) and fast twitch (mATPase $\mathrm{pH}$ 4.6) fibers predominance. D-E-F Short ischemia and prolonged reperfusion. $\mathrm{NADH}$ staining evidences stronger reactive fibers in comparison to the control group. The fast twitch predominance observed in mATPase staining is conserved, and changes in the shape and size (circle) are evident in the HE. G$\mathrm{H}-\mathrm{I}$ Prolonged ischemia and short reperfusion. Smaller fibers are observed and there is an increasing in their surrounding connective tissue. Leukocytes are also present (circle) J-K-L Control group. Normal characteristics in the soleus muscle observed on each staining. Oxidative (NADH) and slow fast twitch (mATPase pH4.6) fibers predominance. $\mathrm{M}-\mathrm{N}-\mathrm{O}$ Short ischemia and prolonged reperfusion. A decreased in the reaction of the fibers to NADH is observed. One fast twitch fiber (yellow circle) is present in the microscopic field for mATPase. Smaller fibers (black circle) are observed in $\mathrm{HE}$ in comparison to the control group. P-Q-R Prolonged ischemia and short reperfusion. There is a disorganization of the cytoplasm observed in the three staining. More than one fast twitch fiber (yellow circle) is present in the microscopic field for mATPase. Magnification 40x. Scale Bar: $25 \mu$. 
Citation: Salazar N, Rosero DH (2017) Recovery of Skeletal Striated Muscle Tissue in a Model of Reversible Injury. Biol Med (Aligarh) 9: 411. doi:10.4172/0974-8369.1000411

Page 4 of 7

\begin{tabular}{|c|c|c|c|c|c|c|}
\hline \multicolumn{7}{|c|}{ Distribution area of fiber type (ums) } \\
\hline \multirow{2}{*}{ Parameters/muscles } & \multicolumn{3}{|c|}{ Extensor carpi radialis longus $(\tilde{\mathbf{x}})$} & \multicolumn{3}{|c|}{ Soleus ( $\tilde{\mathbf{x}})$} \\
\hline & Control & $\begin{array}{l}\text { Reperfusion after } \\
\text { Ischemia 1h }\end{array}$ & $\begin{array}{l}\text { Reperfusion after } \\
\text { Ischemia } 3 \mathrm{~h}\end{array}$ & Control & $\begin{array}{l}\text { Reperfusion after } \\
\text { Ischemia } 1 \mathrm{~h}\end{array}$ & $\begin{array}{l}\text { Reperfusion after } \\
\text { Ischemia } 3 \mathrm{~h}\end{array}$ \\
\hline Oxidative (NADH-TR) & 9263 & 14237.3 & 13009.4 & 36212 & 27046.7 & 11912.3 \\
\hline Glycolytic (NADH-TR) & 30552 & 11038.7 & 11312.4 & 0 & 5327.27 & 5327.84 \\
\hline Slow twitch (mATPase $\mathrm{pH} 4,6$ ) & 1051 & 3178.48 & 4001.7 & 9076 & 21237.92 & 21686.37 \\
\hline Fast twitch (mATPase $\mathrm{pH} 4,6$ ) & 8125 & 15140,04 & $76,92,486$ & 0 & 1591,19 & 393,69 \\
\hline
\end{tabular}

Table 1: Note the total tendency of the areas of each type of fibers in both muscles in comparison to the control groups.

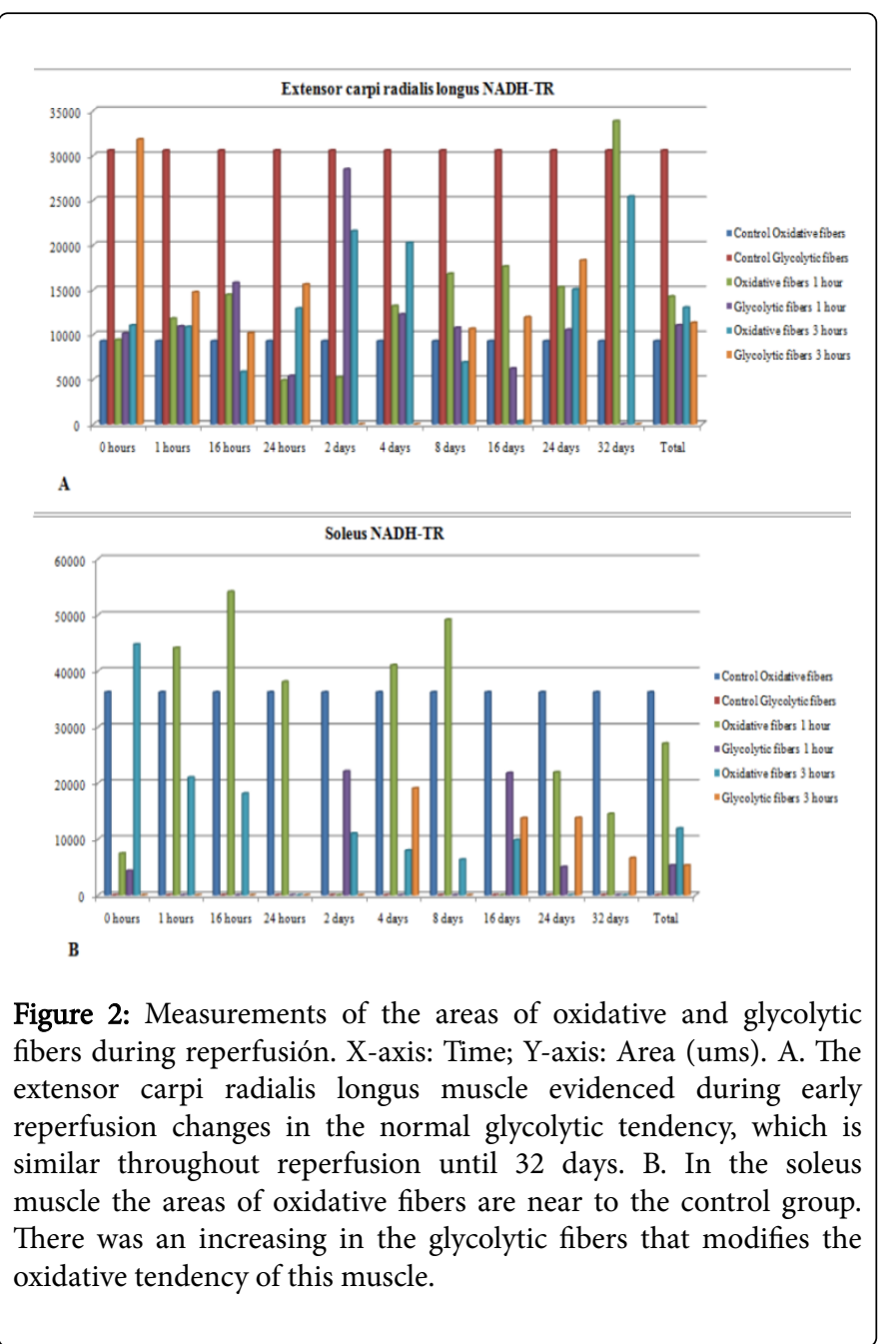

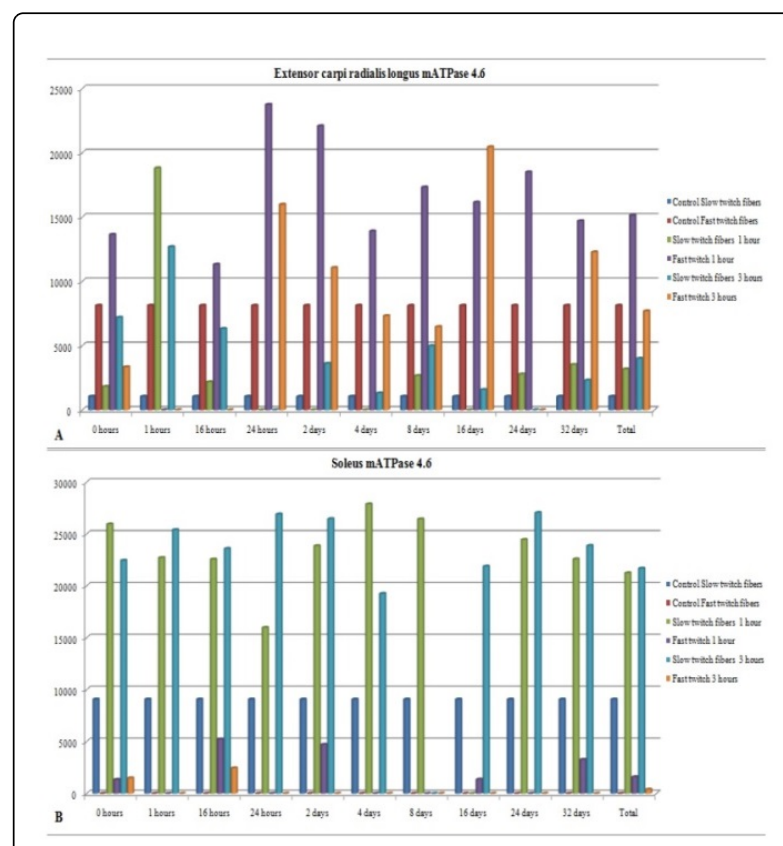

Figure 3: Measurements of the areas of slow twitch fibers and fast twitch fibers during reperfusión. X-axis: Time; Y-axis: Area (ums). A. The extensor carpi radialis longus muscle evidenced a general increasing of the slow twitch fibers, while fast twitch fibers were not severely affected during reperfusion after the ischemic conditions of one hour and three hours. B. The soleus muscle conserved a predominance of slow twitch fibers throughout reperfusion. Nonetheless, the presence of fast twitch fibers was observed during reperfusion after one and three hours of ischemia. 


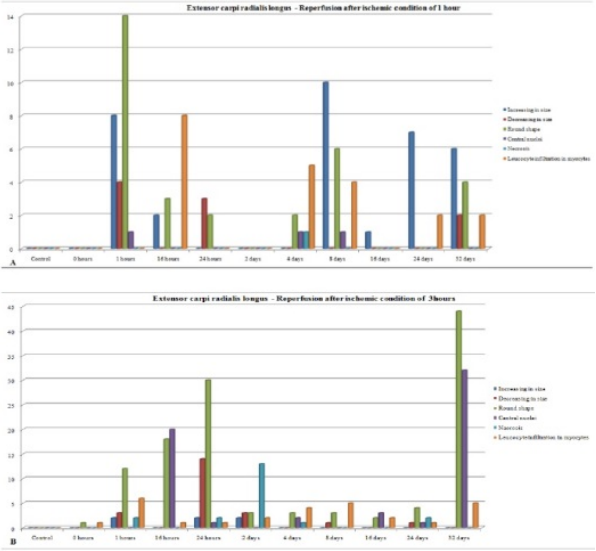

Figure 4: Measurements of abnormal parameters observed in muscle fibers during reperfusión. X-axis: Time; Y-axis: Number of fibers. A. The shape and size of the myocytes are the parameters mainly altered during prolonged reperfusion after one hour of ischemia. B. Throughout reperfusion after three hours of ischemia the measured parameters are present. The increase in fibers with round shape and fibers with central nuclei are notable during early and prolonged reperfusion.

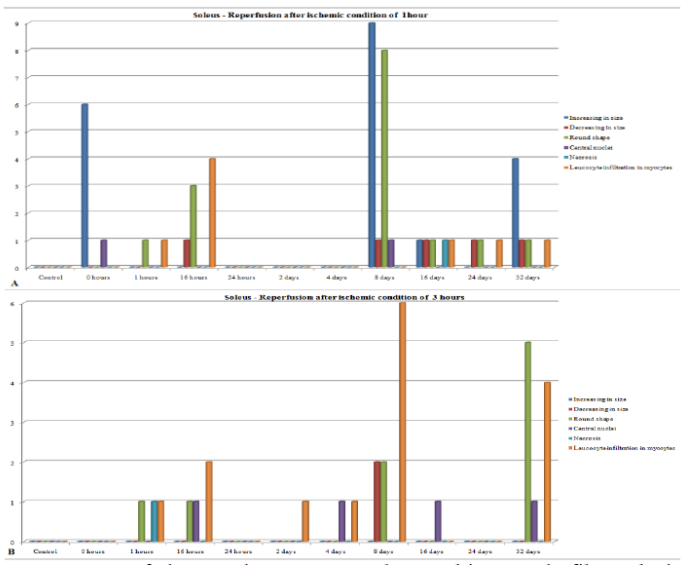

Figure 5: Measurements of abnormal parameters observed in muscle fibers during reperfusión. X-axis: Time; Y-axis: Number of fibers. (A) The increasing in the size of fibers and round shape are the notable changes along reperfusion after one hour of ischemia. (B) During reperfusion after three hours of ischemia round shape and leukocyte infiltration in myocytes are the patterns still present in the prolonged periods.

\section{Discussion}

The recovery of striated muscle tissue undergone reversible injury, such as short ischemic conditions, entails inflammation, changes in metabolic substrates and decreased in ATP levels, among other factors $[19,26]$. Upon releasing the stimulus that caused ischemia, the blood flow is present and injury due to reperfusion occurs [27]. Short periods of reperfusion can cause apparently reversible lesions. Carmo-Araujo et al. described the effects of induced ischemia of four hours in soleus, followed by one hour of reperfusion. The main changes resemble the findings of the present study that include altered shape of the myocytes, decreasing in size and increasing in the space occupied by the connective tissue [5].

Our previous studies evidenced changes in the areas occupied by the muscle fibers and the intramuscular extracellular matrix $[7,8]$. Both patterns have a different tendency in both muscles. At day 32 of reperfusion, the area of the connective tissue measured in the extensor carpi radialis longus was superior to the area of the myocytes. In contrast, at the same day, the area of the connective tissue measured in the soleus muscle was inferior in comparison to the control group [8]. These findings suggest that the increasing in the muscle fibers leads the muscle to maintain its contractile function. These results are consistent to the studies that describe a better recovery in the soleus muscle than in glycolytic predominance muscles [4].

Skeletal muscles contain a high proportion of a determined type of fiber depending on their function and location. For instance, the predominance of extensor muscles is of type IIb fibers, whereas flexor muscles are of oxidative fibers [28,29]. Each kind of muscle fibers respond in a different way when underwent to ischemia and reperfusion injury. Chan et al. observed injury due to three hours of reperfusion in type 2 fibers that endured two hours of ischemia [30]. Likewise, studies conducted by Walters et al. demonstrated that skeletal muscles with oxidative predominance tolerate prolonged periods of ischemia and reperfusion. Their results determined that one of the contributing factors in the damage and the recovery of the muscle fibers is their predominance in the muscle [4]. This characteristic is associated with the strong ability of the soleus muscle to adapt to reperfusion injury [4].

The research published by Sassoli and co-workers considered cell differentiation and function of connective tissue cells in the expression of matrix metalloproteinases [31]. Ghaly and Marsh described an increasing in the concentration of leukocytes, myeloperoxidase, and chemotactic factors, during early hours of reperfusion [32]. A gradual decreasing is present after three days of reperfusion, with a rising in monocyte infiltration and oxidative activity of macrophages during the following periods [32]. The type of fibers and the intramuscular connective tissue influence the recovery of skeletal muscle during reperfusion [33,34]. In this research, although there were inflammatory changes and presence of leukocytes during reperfusion, there was no evidence of fibrosis that led to a replacement of muscle fibers by connective tissue.

The parameters of regeneration as central nuclei are one of the interesting findings still present in prolonged reperfusion up to day 32 . Itoh et al. evidenced central nuclei in the tibialis anterior that was underwent to one hour of ischemia with a gradual decreasing until day 14 of reperfusion [35]. Saclier et al. used the term regenerated myofiber, to refer to the myocytes with central nuclei [36]. The presence of central nuclei indicates a stage of differentiation and recovery in the striated skeletal muscle tissue underwent to injury $[35,36]$. In the present research, the soleus and the extensor carpi radialis longus evidenced different patterns of regeneration for each muscle. Likewise, both muscles evidenced distinct changes in size and shape of muscle fibers, possibly related with oedema and protein degradation after short periods of ischemia, as have seen in muscle fibers obtained from postsurgical patients [37]. 
The follow up after clinical procedures that require the use of the tourniquet are mainly focused on the possible complications and the treated region, such as the joints [38]. Based on the present study, the structures under the level of compression, for example the muscles, are suitable to be also included in this follow up and upcoming therapies. The muscles are also susceptible to the ischemic and reperfusion conditions, so they evidence changes during the post-surgical recovery that can influence the final functional outcomes of patients, being this aspect the main reason for a more complete follow up.

This research provided a histopathological evaluation of skeletal striated muscle tissue during prolonged reperfusion. The evaluated patterns have influence in the postsurgical recovery and explain the impairment in muscle function that occurs in several clinical cases. Based on the findings of the present research up to day 32 of reperfusion, abnormal patterns are still present in muscle fibers mainly in the extensor carpi radialis longus, which are aspects to consider in the postoperative and/or post-traumatic follow up of patients.

\section{Conclusion}

Injury after short ischemic conditions up to three hours can alter the normal characteristics of the skeletal striated muscle tissue in the rat model established in this study. During early and prolonged reperfusion, the affected muscles evidence patterns of adaptation to endure damage due to ischemia, but mainly due to reperfusion. One of them is the predominance of the muscle fibers that change throughout reperfusion to maintain the contractile function of the muscle.

\section{Conflict of Interest}

The authors declared no potential conflicts of interest with respect to the research or publication of this article.

\section{Acknowledgements}

The authors want to acknowledge to the Laboratory of Histology of Universidad del Valle, Teblami Research Group, Nhora Holguín, Martha Ceballos and Henry Vidal of Universidad del Valle, for their valuable contributions to this study.

\section{References}

1. Hocker AD, Boileau RM, Lantz BA, Jewett BA, Gilbert JS, et al. (2013) Endoplasmic Reticulum Stress Activation during Total Knee Arthroplasty. Physiol Rep 1: e00052.

2. Estebe JP, Davies JM, Richebe P (2011) The pneumatic tourniquet: mechanical, ischaemia-reperfusion and systemic effects. Eur J Anaesthesiol 28: 404-11.

3. Wang T, Zhou YT, Chen XN, Zhu AX (2014) Putative role of ischemic postconditioning in a rat model of limb ischemia and reperfusion: Involvement of hypoxia-inducible factor-1a expression. Braz J Med Biol Res 47: 738-45.

4. Walters TJ, Kragh JF, Baer DG (2008) Influence of fiber-type composition on recovery from tourniquet-induced skeletal muscle ischemiareperfusion injury. Appl Physiol Nutr Metab 33: 272-81.

5. Carmo-Araújo EM, Dal-Pai-Silva M, Dal-Pai V, Cecchini R, Anjos Ferreira AL (2007) Ischaemia and reperfusion effects on skeletal muscle tissue: morphological and histochemical studies. Int J Exp Pathol 88: 147-54.

6. Rácz IB, Illyés G, Sarkadi L, Hamar J (1997) The functional and morphological damage of ischemic reperfused skeletal muscle. Eur Surg Res 29: 254-63.
7. Rosero Salazar DH, Salazar Monsalve L (2015) Digital image analysis of striated skeletal muscle tissue injury during reperfusion after induced ischemia. Proc SPIE 9287, 10th International Symposium on Medical Information Processing and Analysis, 92870I

8. Rosero Salazar DH, Elvira Flórez LJ (2016) Image analysis in Gomori's trichrome stain of skeletal muscles subjected to ischemia and reperfusion injury. Exploratory Animal and Medical Research 6: 15-25.

9. Charles AL, Guilbert AS, Guillot M, Talha S, Lejay A, et al. (2017) Muscles susceptibility to ischemia-reperfusion injuries depends on fiber type specific antioxidant level. Front Physiol 8: 52.

10. Rosero-Salazar DH (2016) Image analysis of oxidative and glycolytic muscle fibers during reperfusion injury by segmentation based on regions. International Journal of Morphology 34: 127-135.

11. Chachques JC, Fabiani JN, Perier P, Swanson J, Dreyfus G, et al. (1985) Reversibility of muscular ischemia: a histochemical quantification by the nitroblue tetrazolium (NBT) test. Angiology 36: 493-499.

12. Petrasek PF, Homer-Vanniasinkam S, Walker PM (1994) Determinants of ischemic injury to skeletal muscle. J Vasc Surg 19: 623-31.

13. Vaillancourt C, Shrier I, Vandal A, Falk M, Rossignol M, et al. (2004) Acute compartment syndrome: how long before muscle necrosis occurs? CJEM 6: 147-154.

14. Dumont NA, Bentzinger CF, Sincennes MC, Rudnicki MA (2015) Satellite cells and skeletal muscle regeneration. Compr Physiol 5: 1027-1059.

15. Corona BT, Rathbone CR (2014) Accelerated functional recovery after skeletal muscle ischemia-reperfusion injury using freshly isolated bone marrow cells. J Surg Res 188: 100-109.

16. Vignaud A, Hourde C, Medja F, Agbulut O, Butler-Browne G, et al. (2010) Impaired skeletal muscle repair after ischemia-reperfusion injury in mice. J Biomed Biotechnol 2010: 724914.

17. Institute for Laboratory Animal Research (2010) International guidelines for care and use of animals (8th edn.). The National Academies Press, Washington D.C.

18. Rodrigues AC, Silva M dal D, Pai VD (1994) Distribution of different fibre types of M. extensor carpi radialis longus of the rat. Anat Histol Embryol 23: 352-356.

19. Carvalho AJ, McKee NH, Green HJ (1997) Metabolic and contractile responses of fast and slow twitch rat skeletal muscles to ischemia and reperfusion. Plast Reconstr Surg 99: 163-171.

20. Carvalho AJ, Hollett P, McKee NH (1995) Recovery of synergistic skeletal muscle function following ischemia. J Surg Res 59: 527-533.

21. Woitaske MD, McCarter RJ (1998) Effects of fiber type on ischemiareperfusion injury in mouse skeletal muscle. Plast Reconstr Surg 102: 2052-2063.

22. Latorre R, Gil F, Vasquez J (1993) Skeletal muscle fiber types in the dog. I Anat 182: 329-337.

23. Kalmar B, Blanco G, Greensmith L (2012) Determination of muscle fiber type in rodents.

24. Mehmood I, Ejaz N, Sajjad M, Baik SW (2013) Prioritization of brain MRI volumes using medical image perception model and tumor region segmentation. Comput Biol Med 43: 1471-1483.

25. Young I, Gerbrands J, van Vliet L (1998) Fundamentals of image processing (2nd edn.). Technology Duo, Delf University of Technology, Netherlands. p. 112.

26. Carvalho AJ, McKee NH, Green HJ (1996) Metabolic and contractile responses of fast- and slow-twitch rat skeletal muscles to ischemia. Can J Physiol Pharmacol 74: 1333-41.

27. Carden DL, Granger DN (2000) Pathophysiology of ischaemiareperfusion injury. J Pathol 90: 255-66.

28. Lexell J, Jarvis JC, Currie J, Downham DY, Salmons S (1994) Fibre type composition of rabbit tibialis anterior and extensor digitorum longus muscles. J Anat 185: 95-101.

29. Soukup T, Zacharová G, Smerdu V (2002) Fibre type composition of soleus and extensor digitorum longus muscles in normal female inbred Lewis rats. Acta Histochem 104: 399-405. 
Citation: Salazar N, Rosero DH (2017) Recovery of Skeletal Striated Muscle Tissue in a Model of Reversible Injury. Biol Med (Aligarh) 9: 411. doi:10.4172/0974-8369.1000411

Page 7 of 7

30. Chan RK, Austen WG, Ibrahim S, Ding GY, Verna N, et al. (2004) Reperfusion injury to skeletal muscle affects primarily type II muscle fibers. J Surg Res 122: 54-60.

31. Sassoli C, Nosi D, Tani A, Chellini F, Mazzanti B, et al. (2014) Defining the role of mesenchymal stromal cells on the regulation of matrix metalloproteinases in skeletal muscle cells. Experimental Cell Research 323: 297-313.

32. Ghaly A, Marsh DR (2010) Ischaemia-reperfusion modulates inflammation and fibrosis of skeletal muscle after contusion injury. Int J Exp Pathol 91: 244-55.

33. Purslow PP (2010) Muscle fascia and force transmission. J Bodyw Mov Ther 14: 411-417.

34. Turrina A, Martínez-González MA, Stecco C (2013) The muscular force transmission system: role of the intramuscular connective tissue. J Bodyw Mov Ther 17: 95-102.
35. Itoh M, Kudoh Y (2011) Morphologic changes in rat skeletal muscle after anterior tibial muscle and artery ligation. Journal of Clinical Welfare 8: 56-63.

36. Saclier M, Cuvellier S, Magnan M, Mounier R, Chazaud B (2013) Monocyte/macrophage interactions with myogenic precursor cells during skeletal muscle regeneration. FEBS J 280: 4118-30.

37. Jawhar A, Hermanns S, Ponelies N, Obertacke U, Roehl H (2015) Tourniquet-induced ischaemia during total knee arthroplasty results in higher proteolytic activities within vastus medialis cells: a randomized clinical trial. Knee Surg Sports Traumatol Arthrosc 24: 3313-21.

38. Alcelik I, Pollock RD, Sukeik M, Bettany-Saltikov J, Armstrong PM, et al. (2012) A comparison of outcomes with and without a tourniquet in total knee arthroplasty: a systematic review and meta-analysis of randomized controlled trials. J Arthroplasty 27: 331-340. 